\title{
Comparison of Commonly Used Image Interpolation Methods
}

\author{
Dianyuan Han \\ Dept. of Computer Engineering \\ Wei Fang University \\ Shandong 261061, China \\ wfhdy@163.com
}

\begin{abstract}
Image magnification algorithms directly affect the quality of image magnification. In this paper, based on the image interpolation algorithm principle, features of the nearest neighbor interpolation, bilinear interpolation, bicubic interpolation and cubic $B$ spline interpolation were analyzed. At the same time, their advantages and disadvantages were compared. In the experiment, image magnification performance of different interpolation algorithms was compared from subjective and objective aspects. The experimental results give the guidance for the user to choose a suitable algorithm to achieve optimum results according to different application.
\end{abstract}

Keywords- Image magnification; Interpolation algorithm; Performance comparison

\section{INTRODUCTION}

Image magnification is one of the basic image operations, and is widely used in many applications[1]. The image magnification is a conversion process from a low resolution image to a high resolution image. The image magnification is essentially image interpolation process. There have been a lot of practical image magnification methods which have their own characteristics, advantages and disadvantages. The choice of the different image magnification algorithm directly affects enlarged image quality, so it is important to find a suitable algorithm to improve the image magnification quality.

In current, the commonly used image magnification method is interpolation method. In the experiment, image magnification performance of different interpolation algorithms was compared from subjective and objective aspects to give the guidance for the user to choose a suitable algorithm to achieve optimum results according to different application.

\section{IMAGE AMPLIFICATION PRINCIPLE}

The basic principle of image magnification is to increase the image pixel number, so a low resolution image is converted to a high resolution image.

When a small image is enlarged, for example if an image is zoomed to $400 \%$ shown in Fig. 1., the color values of original 4 adjacent pixels marked A, B, C, and D in (a) were filled in the new A, B, C, and D locations in (b) accordance with the magnification factor. But there are a large number of pixels which values are unknown between A, B, C, and D, such as P. so the values of these pixels should be calculated throw interpolating estimation.

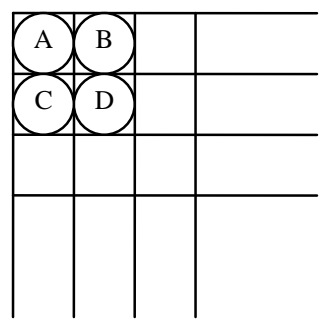

(a)

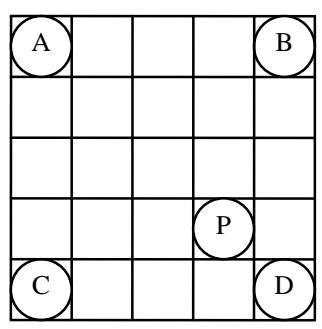

(b)
Figure 1. Schematic diagram of image magnification

\section{COMMON INTERPOLATION ALGORITHM}

\section{A. Nearest neighbor interpolation}

In nearest neighbor interpolation algorithm, the position of pixel $\mathrm{P}$ in the magnified image is converted into the original image, and the distance between $\mathrm{P}$ and its neighbor points $\mathrm{A}, \mathrm{B}, \mathrm{C}$ and $\mathrm{D}$ were calculated. Then the color values of pixel $P$ was set as the values of the pixel which was nearest to $\mathrm{P}[2]$.

In Fig. 2, suppose $(i, j),(i, j+1),(i+1, j)$ and $(i+1, j$ $+1)$ are the 4-neighbor points, and there values are $f(i, j)$, $f(i, j+1), f(i+1, j)$ and $f(i+1, j+1)$. The distance between $(u, v)$ and $(i, j),(i, j+1),(i+1, j)$ and $(i+1, j+1)$ were calculated, then the values of $(u, v)$ was set as the value of the point which is nearest to $(\mathrm{u}, \mathrm{v})$. [3]

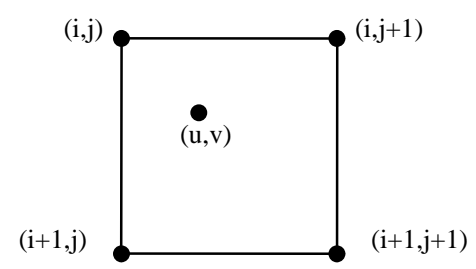

Figure 2. Diagram of nearest neighbor interpolation algorithm

\section{B. Bilinear interpolation}

In bilinear interpolation algorithm[4], the position of pixel $\mathrm{P}$ in the magnified image was converted into the 
original image, then the Influence of the four pixel points A, $\mathrm{B}, \mathrm{C}$ and $\mathrm{D}$ was calculate. The nearer distance to the point $\mathrm{P}$, the value is greater, which indicates the greater effects. The diagram of bilinear interpolation is shown in Fig. 3.

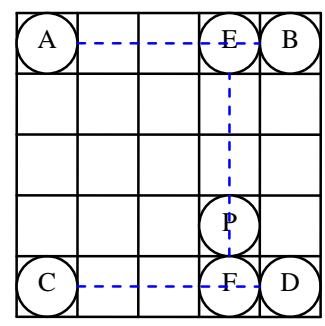

Figure 3. Diagram of bilinear interpolation algorithm

Suppose the coordinate of A, B, C and D are ( $i, j),(i, j$ $+1),(i+1, j)$ and $(i+1, j+1)$. The coordinate of $P$ is $(u, v)$. The bilinear interpolation algorithm consists of three steps:

Step 1: to calculate the influence of A and B and denote it as E.

$f(i, j+v)=[f(i, j+1)-f(i, j)] v+f(i, j)$

Step 2: to calculate the influence of $\mathrm{C}$ and $\mathrm{D}$ and denote it as $\mathrm{F}$.

$$
f(i+1, j+v)=[f(i+1, j+1)-f(i+1, j)] v+f(i+1, j)
$$

Step 3: to calculate the influence of $\mathrm{E}$ and $\mathrm{F}$ and denote it as $\mathrm{P}$.

$$
\begin{aligned}
& f(i+u, j+v)=(1-u)(1-v) f(i, j)-(1-u) v f(i, j+1) \\
& +u(1-v) f(i+1, j)+u v f(i+1, j+1)
\end{aligned}
$$

\section{Bicubic Interpolation}

Bicubic interpolation is similar to bilinear interpolation algorithm. For the unknown pixel $\mathrm{P}$ in amplified image, its influence sphere is expanded to its 16 adjacent pixels, then the color value of $\mathrm{P}$ is calculated by these 16 pixels according to their distance to $\mathrm{P}$. The diagram of bicubic interpolation algorithm is shown in Fig.4.

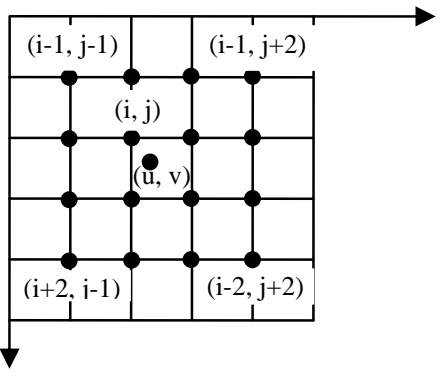

Figure 4. Diagram of bicubic interpolation algorithm

Compared with bilinear interpolation algorithm, bicubic interpolation algorithm extends the influence with more points, and uses advanced interpolation algorithm.

In the horizontal direction, to calculate the value of e between two points $A$ and $B$, it is need to use four pixels value of $\mathrm{A}, \mathrm{B}, \mathrm{A}-1, \mathrm{~B}+1$ and get a smooth curve through a nonlinear calculation. The diagram of nonlinear interpolation algorithm is shown in Fig.5.

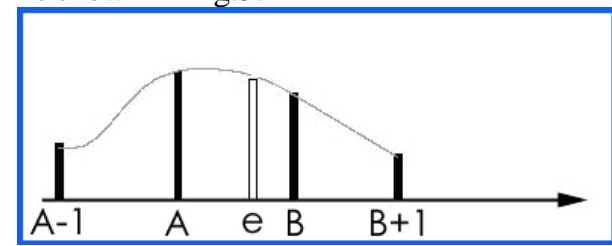

Figure 5. Diagram of nonlinear interpolation algorithm

On the basis of calculating the lateral interpolation effects, the operation as mentioned above is extended into two dimensional space to calculate the longitudinal interpolation effects.

\section{Cubic B-SPline}

In order to get better image quality, many new algorithms have been proposed, they use more complex improved interpolation method such as B spline [5] and Mitchell interpolation algorithm. Their objective is to make the interpolation curve more smoothly and the image edge more perfect.

The function of cubic B-spline is as follow[2].

$$
f(x)=\sum_{k=-\infty}^{+\infty} B_{k, n+1}(x) \bullet f\left(x_{k}\right)
$$

Three order B-spline function is as follows:

$$
B_{i, 3}=\left\{\begin{array}{cl}
\frac{\left(x-x_{i}\right)^{2}}{\left(x_{i+1}-x_{i}\right)\left(x_{i+2}-x_{i}\right)} & , x_{i} \leq x \leq x_{i+1} \\
\frac{\left(x-x_{i}\right)^{2}}{\left(x_{i+1}-x_{i}\right)\left(x_{i+2}-x_{i}\right)}-\frac{\left(x_{i+2}-x\right)\left(x-x_{i+1}\right)}{\left(x_{i+2}-x_{i+1}\right)\left(x_{i+3}-x_{i+1}\right)} & , x_{i+1} \leq x \leq x_{i+2} \\
\frac{\left(x-x_{i+3}\right)^{2}}{\left(x_{i+3}-x_{i+1}\right)\left(x_{i+3}-x_{i+2}\right)} & , x_{i+2} \leq x \leq x_{i+3} \\
0 & \text { everywhere }
\end{array}\right.
$$

\section{EXPERIMENT RESULT}

In order to test the performance of the algorithm, We have developed a MATLAB 2009[6] implementation on Intel(R) Core(TM) 2 T7200 2.00GHz CPU, 2.0GB RAM computer.

To compare the performance of the different algorithm, a test image was zoomed out to half of the original size. And then the shrunken image was expanded to its original size by using different algorithm.

At last the enlarged image quality was compared From two aspects: subjective and objective.

\section{A. Objective Evaluation}

In this paper, the mean square signal-to-noise ratio (SNR) was used to evaluate image expansion performance of different algorithms.

Let $\mathrm{f}(\mathrm{x}, \mathrm{y})$ represents the original image with the size of $\mathrm{M} \times \mathrm{N}, \hat{f}(x, y)$ represents the enlarged image which has been zoomed out to half size of its original image, and 
$\bar{f}$ represents the mean gray value of the original image. $\bar{f}$ is defined as fellow.

$$
\bar{f}=\frac{1}{M N} \sum_{x=0}^{M-1} \sum_{y=0}^{N-1} f(x, y)
$$

SNR is defined as (7).

$$
S N R=10 \lg \left\{\frac{\sum_{x=0}^{M-1} \sum_{y=0}^{N-1}[f(x, y)-\bar{f}]^{2}}{\sum_{x=0}^{M-1} \sum_{y=0}^{N-1}[\hat{f}(x, y)-f(x, y)]^{2}}\right\}
$$

In the experiment, we selected 30 images representative image to test, the results is shown in Tab.1.

TABLE I. Contrast table of SNR with different interpolation mothods

\begin{tabular}{ccccc}
\hline $\begin{array}{c}\text { Tested } \\
\text { Image }\end{array}$ & $\begin{array}{c}\text { Nearest } \\
\text { Neighbour }\end{array}$ & Bilinear & Bicubic & $\begin{array}{c}\text { Cubic } \\
\text { B-SPline }\end{array}$ \\
\hline image 1 & 19.1112 & 23.3023 & 23.4204 & 22.5028 \\
image 2 & 16.0059 & 18.4308 & 18.5899 & 17.9319 \\
image 3 & 20.0614 & 25.3736 & 25.6634 & 24.3505 \\
image 4 & 15.9497 & 18.7056 & 18.9392 & 18.1074 \\
image 5 & 17.1252 & 20.2387 & 20.9872 & 18.6582 \\
image 6 & 18.15564 & 22.137185 & 22.24938 & 21.37766 \\
image 7 & 14.40531 & 16.58772 & 16.73091 & 16.13871 \\
image 8 & 17.05219 & 21.56756 & 21.81389 & 20.697925 \\
image 9 & 12.75976 & 14.96448 & 15.15136 & 14.48592 \\
image 10 & 12.8439 & 15.179025 & 15.7404 & 13.99365 \\
\hline
\end{tabular}

SNR can reflect the magnified image quality, the larger SNR means the higher image quality.

It can be seen from the above table that the SNR between the original image and the magnified image get from the nearest neighbour interpolation is the minimum, and bilinear interpolation algorithm is much larger, bicubic interpolation is the largest, and the B spline larger than the nearest neighbor method but less than the bilinear interpolation algorithm and bicubic interpolation method.

The complexity of Different interpolation algorithm is different, and their average running time also has bigger difference. The average run time is shown in Fig.6.

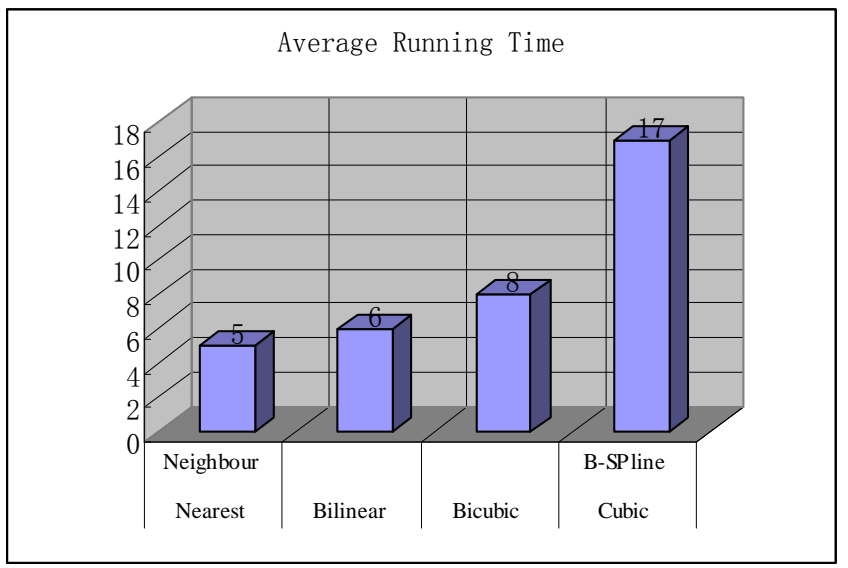

Figure 6. Average running time of different interpolation algorithm

\section{B. Subjective Evaluation}

Image is used to be watch by people, so it is more suitable evaluate the magnified image quality with subjective methods.

In this paper, 20 observers were invited to observe magnified images and give evaluation. At last the image scoring average and used to evaluate the subjective image quality.

The subjective evaluations with different interpolation methods are shown in Tab. 2.

TABLE II. Contrast table of subjective evaluation with different

\begin{tabular}{|c|c|c|c|c|}
\hline $\begin{array}{c}\text { Interpolation } \\
\text { Type }\end{array}$ & $\begin{array}{l}\text { Subjective } \\
\text { Feelings }\end{array}$ & $\begin{array}{c}\text { Image } \\
\text { Contour }\end{array}$ & $\begin{array}{c}\text { Overall } \\
\text { Evaluation }\end{array}$ & $\begin{array}{c}\text { Processing } \\
\text { Time } \\
\text { (seconds) }\end{array}$ \\
\hline $\begin{array}{l}\text { Nearrst_- } \\
\text { Neighbour }\end{array}$ & $\begin{array}{c}\text { obvious } \\
\text { mosaic } \\
\text { phenomenon }\end{array}$ & Not clear & Worst & 5 \\
\hline Bilinear & $\begin{array}{l}\text { blur, not } \\
\text { sharp }\end{array}$ & $\begin{array}{c}\text { not clear, } \\
\text { serrate } \\
\text { phenomenon }\end{array}$ & Poor & 6 \\
\hline Bicubic & $\begin{array}{l}\text { fuzzy, } \\
\text { sharper }\end{array}$ & $\begin{array}{c}\text { serrate } \\
\text { phenomenon } \\
\text { has } \\
\text { improved }\end{array}$ & Better & 8 \\
\hline B-Spline & $\begin{array}{l}\text { relatively } \\
\text { clear, sharp }\end{array}$ & $\begin{array}{c}\text { Edge } \\
\text { becomes } \\
\text { clear, serrate } \\
\text { phenomenon } \\
\text { disappeared }\end{array}$ & Good & 17 \\
\hline
\end{tabular}
interpolation mothods

An example of image magnification is shown in Fig.7. 

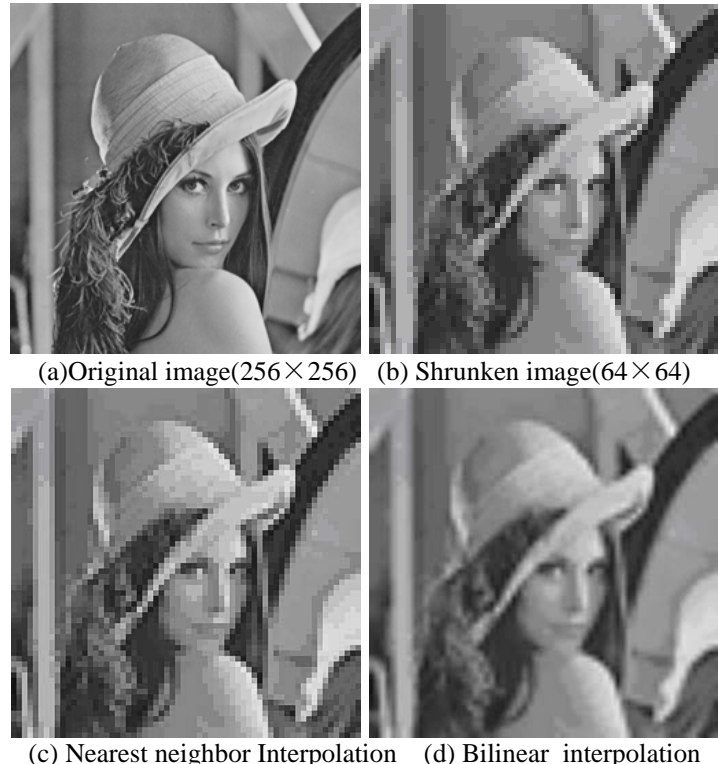

(c) Nearest neighbor Interpolation (d) Bilinear interpolation

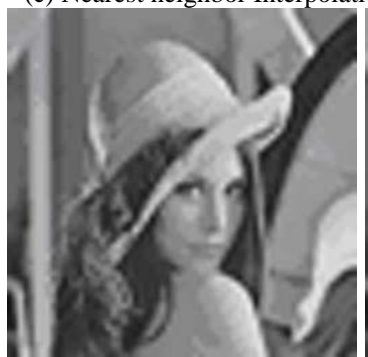

(e) Bicubic interpolation

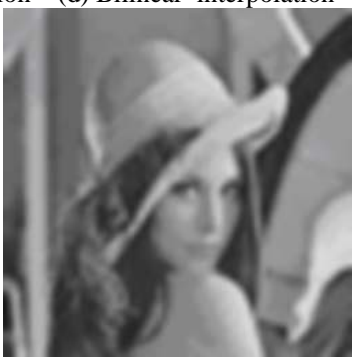

(f) Cubic B-SPline interpolation

Figure 7. Diagram of nonlinear interpolation algorithm

\section{CONCLUSIONS}

Nearest neighbour interpolation algorithm is the most simple and fast algorithm. It has the advantages of fast speed, but it can bring significant distortion and it will appear mosaic and saw tooth phenomenon.

Bilinear interpolation method is more complex than the nearest neighbour method, and so it has larger calculation. It has no gray discontinuity defects and has satisfactory results. This method has a low pass filtering properties, so that the high frequency component is faded and the image contour has some degree of fuzzy. As the results of bilinear

interpolation algorithm is continuous, so the visual effect is better than the nearest point interpolation algorithm, but the operation speed is slightly slower. Because of this, if someone pays more attention to speed and good visual effect, this is a good compromise.

Bicubic interpolation algorithm can get relatively clear picture quality, but it needs larger amount of calculation. This algorithm now is most commonly used in many image processing software such as Photoshop, After Effects, Avid and Final Cut Pro etc.

The magnified image by using of cubic B-spline interpolation algorithm is smooth and has no obvious saw tooth phenomenon. But in the interpolation process it takes the form of a low pass filter and suppress high frequency component in varying degrees. When the amplification factor is higher, it will cause the edge fuzzy and false artificial traces.

The experimental results give the guidance for the user to choose a suitable algorithm to achieve optimum results according to different application.

\section{ACKNOWLEDGMENT}

We acknowledge the financial support of Weifang science and technology development program (NO.20111127) and Shandong province science and technology development program (NO.2011YD01047).

\section{REFERENCES}

[1] van Ouwerkerk J D. "Image super resolution survey". Image and Vision Computing, 2006, 24( 10) : 1039-1052.

[2] Jia Yonghong. "Digital image processing(The Second Edition)".Wu Han China: Wu Han university press, 2010: 114-116.

[3] Zhao haifeng, Zhou Yongfei, Huang Ziqiang. "Comparison of Image Amplifying Method”. Modern Electronics Technique, 2010,(24):3336.

[4] Wang Sen, Yang Kejian. "An image scaling algorithm based on bilinear interpolation with $\mathrm{VC}++$ " . Jou rnal of Techniques of Automation \& Applications, 2008, 27( 7) : 44-45.

[5] Feng Jiefei1, Han Huijian.”Image enlargement based on non-uniform B-spline interpolation algorithm”. Journal of Computer Applications, 2010,30(1):82-84.

[6] Rafael c. Gonzalez, Richard E. Woods. "Digital image processing second Edition”, Beijing: Publishing house of electronics industry,2008, 